\title{
Apparent image rotation in stereoscopic vision: The unbalance of the pupils
}

\author{
GEORG von BEKESY2 \\ UNIVERSITY OF HAWAII
}

\begin{abstract}
In binocular vision the apparent rotation of an object relative to the frontal plane seldom corresponds with the actual rotation. The reason for this is a difference in the brightness of the object in both eyes. It can be produced by a difference in the pupil size, adaptation, or sensitivity. One of the purposes of the pupils seems to be to compensate for this brightness unbalance and to make the apparent rotation come closer to the actual one. Unbalance was produced by placing a density filter before one eye, and the corresponding rotations were then measured. The rotations are affected by the irradiation in the eye, as a physical component, but also by the lateral inhibition in the visual nervous system. If the object and the background have a different color but there is no brightness difference on the edges of the object, there is no distorted rotation.
\end{abstract}

The Three Different Types of Apparent Rotations in a Binocular Microscope

It may be very disturbing to find during surgery done under a binocular preparatory microscope that the actual angle of rotation of an object from the horizontal plane may be misjudged. This is especially disturbing when the surface of an object and the surface of an instrument that are actually parallel seem to be tilted against each other.

The first type of rotation I would like to call static binocular rotation. If we place a piece of paper with a narrow black stripe under the microscope so that the stripe is perpendicular to the axis between the two objectives and focus the objectives and adjust the oculars properly for both eyes, we may see the black stripe in the same plane as the paper. But after longer observations, we may see an apparent rotation around the axis of the stripe. This rotation can be produced by a small difference in the magnification for both eyes (Ogle, 1964) or by a difference in pupil size or sensitivity. It can be seen

Fig. 1. The image of a white stripe on the retina has a slope on the edge of the luminance distribution because of an irradiation in the eye. If a density filter is placed before one eye, the decrease in luminance will narrow the width of the image. during fixed adjustments of the microscope. The rotation disappears for a stripe that parallels the axis of the two objectives. Semiglossy objects can have a large apparent rotation if one objective of the microscope receives more light than the other because of poor illumination.

The second type of apparent rotation, the dynamic, can be monocularly or binocularly seen. It occurs only during focusing of the microscope, when the apparent width of the stripe changes and its edge becomes blurred. It is independent of the direction of the stripe. The same apparent rotation can be obtained if we observe a slot and change its width with a micrometer screw. There is a certain optimal speed for the width change that produces a maximal momentary rotation. Unfortunately, under the binocular microscope that speed is the same speed with which focusing is usually done. For a slit with a width of $5 \mathrm{~mm}$ seen from $25 \mathrm{~cm}$, a change of $\pm 0.3 \mathrm{~mm}$ produced in $1 / 2 \mathrm{sec}$ gives a good rotation.

The rivalry between the visual fields of both eyes can produce the third type of apparent rotation in the binocular microscope. If the focusing of the image in both eyes is not proper or there is a large difference in the illumination pattern of the images in both eyes, we may have difficulty in fusing them. This can occur especially during the observation of a tilted surface under the microscope. If there is no fusion between the two images, there is no depth perception and the object appears flat. This means a rotation from the original tilted position has occurred. Usually both eyes interchange in 2 to $4 \mathrm{sec}$ because of the rivalry, but there are persons in whom one eye dominates for 20 to $40 \mathrm{sec}$.

\section{left eye}

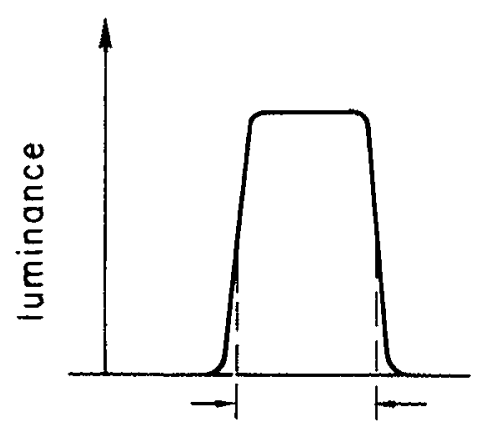

These are the three types of phenomena that make the apparent angle of the surface of an object so seldom coincide with the actual one when seen under a binocular microscope. Of the three, the static type is the most disturbing. Since it occurs also with normal binocular vision, it will be investigated in this paper.

Apparent Rotation of a Vertical Stripe Produced by a Brightness Difference Between Both Eyes

If we let a pendulum bob made from a flat white disk swing in a plane and then place a gray density filter before one eye, we can observe two phenomena. First, the white disk does not seem to swing in a plane but in an ellipse. This is the well-known Pulfrich phenomenon. But besides this, the white disk seems to be rotated along its vertical axis. This rotation of the disk is about the same whether it swings or stands still. It was discussed extensively under Irradiation Stereoscopy by Ogle (1962), though first described by Münster (1941) and later by Cibis and Haber (1951).

There is a purely physical explanation for the rotation. The luminance of the image of a white vertical stripe on a black background (as illustrated in Fig. 1) on the retina does not fall off on the edges immediately. This is so even for a very sharp edge of the white stripe. It is the irradiation occurring in the lens and the vitreous body of the eye that blurs the edges so that the luminance shows a certain slope on the edge, shown for the left eye in Fig. 1. Because of this, a decrease in the luminance of the image of the white stripe by decreasing the size of the pupil or placing a density filter before the eye will reduce the width of the stripe image, as can

$$
\text { right eye }
$$

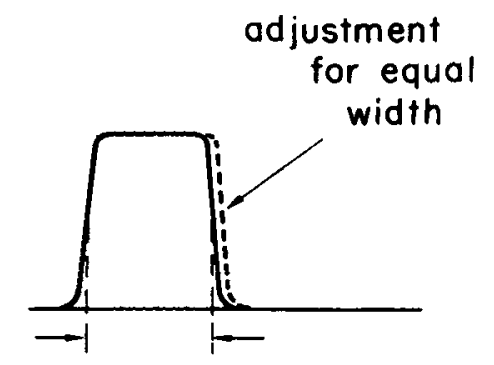




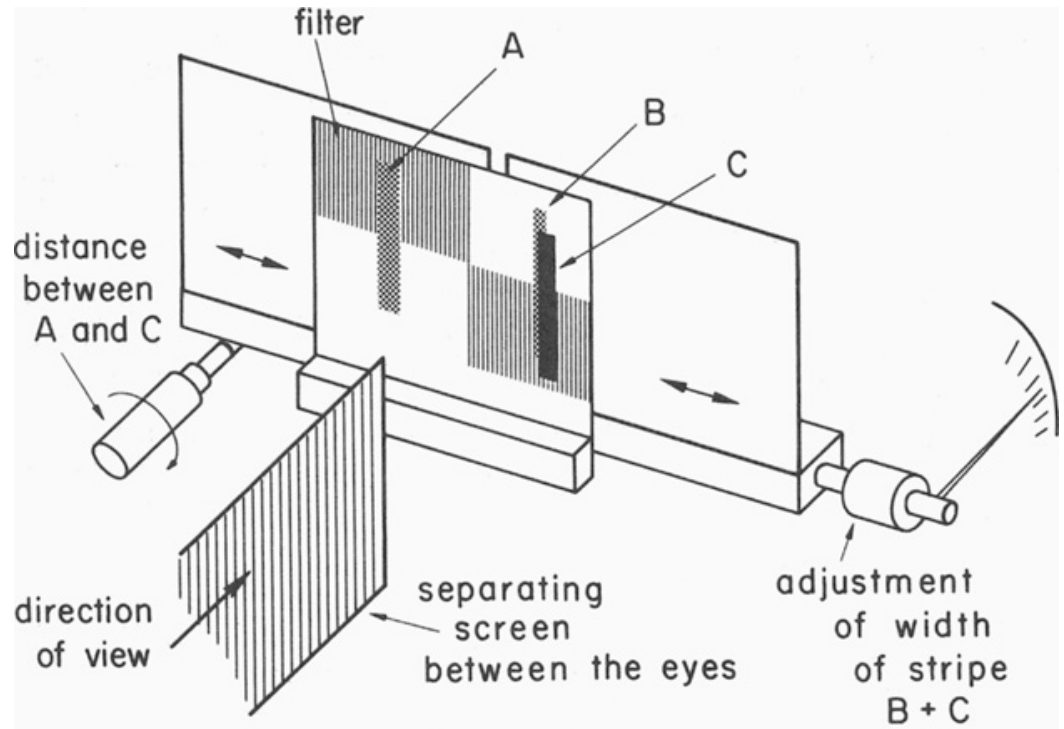

be seen for the right eye in Fig. 1. The same will occur if the threshold of the retina is increased, so that the lower levels of the luminance are no more effective. The binocular fusion of the images on the left and right eye with different widths will produce a rotation.

The slope of the luminance curve as such does not determine the rotation, since it is transformed by the retina into a brightness curve, which in its turn is modified by lateral inhibition. To what degree these three phenomena contribute to the final slope in the sensation magnitude distribution is under discussion.

\section{Compensation for a Rotation by Unilateral} Change in Width

An apparatus similar to that described in Fig. 2 was used to estimate the slope and the decrease in width of the brightness distribution when a density filter is placed

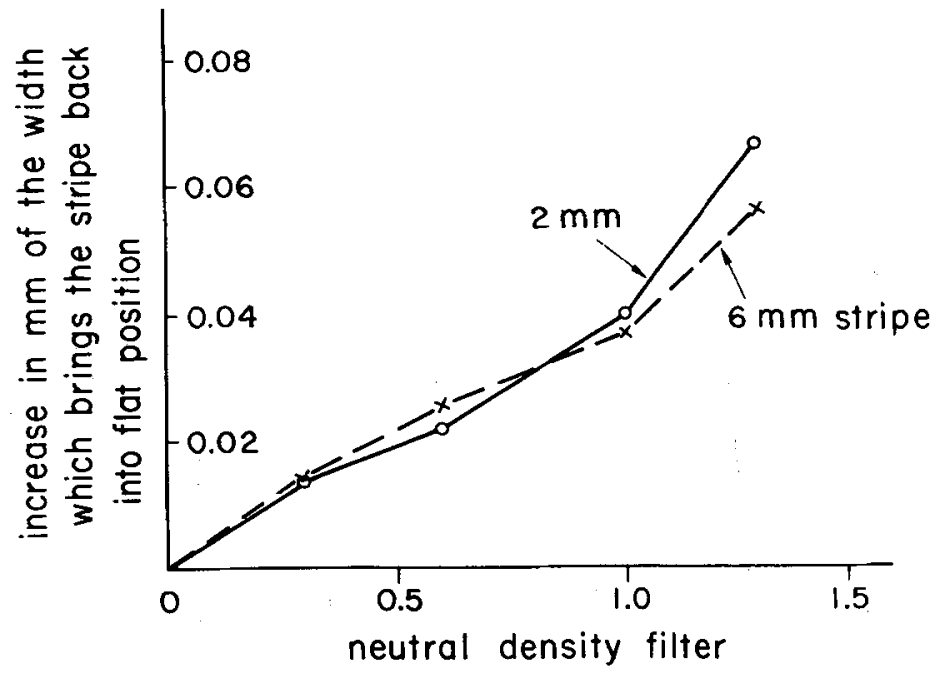

Fig. 2. Apparatus to present one eye with a black stripe, $A$, and the second eye with a black stripe, $B / C$, with adjustable width. If the width of both stripes is equal, the fused image will be flat in front of the O. But if there is a difference, the black stripe will rotate along its vertical axis.

stripe A was also glued on a Plexiglas sheet and moved in the same plane as $B$. The distance between the Plexiglas sheets in front and back was made as small as possible to avoid any type of parallaxis. The apparatus was illuminated from the back and the stripes were seen from $25 \mathrm{~cm}$. It was very important to have the width of the stripes on the upper and lower end exactly the same, since otherwise the stripes show a twist under binocular vision.

Besides a version with white stripes made by windows in a black paper, a model was made with windows in a white paper. The windows were illuminated from the back with one type of light and the surrounding white paper from the front with another light, so that stripes in any surroundings could be produced.

Several precautions were necessary. A headrest and artificial pupils were used. Also, it was necessary in the beginning to place horizontal windows before the eyes so that the ends of the stripes could not be seen, which may influence the magnitude of the rotation. Since the rotation was compensated for during the measurement, this was not too essential. A more difficult problem is that both eyes are not always equally adapted. To avoid this, the Os were asked to look at the windows and surroundings for a while before doing actual observations.

Two equal density filters were placed half across the two openings, as shown in Fig. 2. In this case, the upper part of the stripe will rotate in one direction and the lower part in the opposite direction. The width of the stripe $B / C$ was so adjusted that first the upper part of the window appeared flat and then the lower one. Half the difference in the micrometer reading was plotted in the ordinate of Fig. 3 as the decrease in width produced by a certain density filter. The measurements were done with white stripes seen from $25 \mathrm{~cm}$ and with a luminance of $5 \mathrm{fL}$. Three Os were used for several weeks, and the curves represent the mean values of 10 sets of observations.

Fig. 3. A neutral density filter placed before one eye produces rotation of a black or white stripe that can be compensated by a change in the width of the stripe presented to the eye with the filter. 


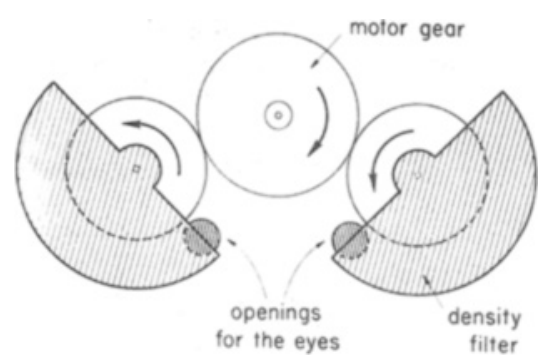

Fig. 4. Since a density filter placed before one eye will change its adaptation and pupil size relative to the other eye, the density filters were exchanged periodically between the two eyes.

The rotation produced by a density filter is inversely proportional to the width of the stripe. But the slope on the edge of the stripe is not influenced by the width of the stripe, as can be seen in Fig. 3, where the two curves for the width of 2 and $6 \mathrm{~mm}$ are close together.

Elimination of the Sensitivity Unbalance of the Eyes

Even with an artificial pupil, a vertical stripe without a density filter can show a rotation. The rotation is caused by a difference in sensitivity in both retinas produced mostly by a difference in adaptation. It was found that this disturbing effect could be much reduced when the density filter was presented to both eyes alternately and the rotation between the two extreme positions was observed.

Figure 4 shows the apparatus used for this purpose. It consisted mainly of two gears that were driven in opposite directions by a motor. On the two gears two celluloid density filters were attached, so that the openings for the two eyes were alternately covered or open. The speed of rotation was one rotation per second. Usually, the $O$ was asked to look through this device for $40 \mathrm{sec}$ before starting observations. If there was still an asymmetry of the apparent rotations present, a sign of a more permanent difference in the sensitivity of the retina, it was then compensated for by changing the diameter of the opening of one of the artificial pupils, which consisted of a small photographic stop.

\section{The Role of the Pupils}

It was always unclear to me why the eye

Fig. 6. There are people with almost frozen pupils (dashed line) whose pupil diamter does not change when looking at a dark or a bright surface. needs a pupil. It is generally accepted that thy pupil performs three main functions (Davson, 1950). (1) The pupil permits the eye to control the exceedingly wide range of luminance in nature and to protect the eye, perhaps, from too much light. But the reaction time of the pupil is relatively slow and the change of the diameter of the opening not large enough to solve this problem. Adaptation seems to be a much better solution. Besides this, many people have pupils that change very little under extreme conditions. (2) The depth of focus is increased by the pupil's contracting its opening in bright light. But this holds for short distances only, since the small focal length of the eye lens gives a great depth of focus for both short and long distances. (3) The aberration of the optical system in bright light is minimized when the pupil is made small.

It is my opinion that besides these three functions, the purpose of the pupils is to equalize the brightness of an object in both eyes, since otherwise we would see the object not in the normal position in space but rotated. This holds for objects close or far away, especially if they are small.

It is possible to obtain a rotation simply by closing one eye for $30 \mathrm{sec}$. This produces an adaptation and pupil unbalance. Unfortunately, this situation holds even if we put a density filter in front of one eye. To illustrate the size of the effect, one eye was covered with a density filter of 1.0 for a certain time interval and the rotation of a 2 -mm-wide vertical stripe was observed as it changed with time. The effect produced by the pupil and adaptation unbalance is shown in

\section{light}

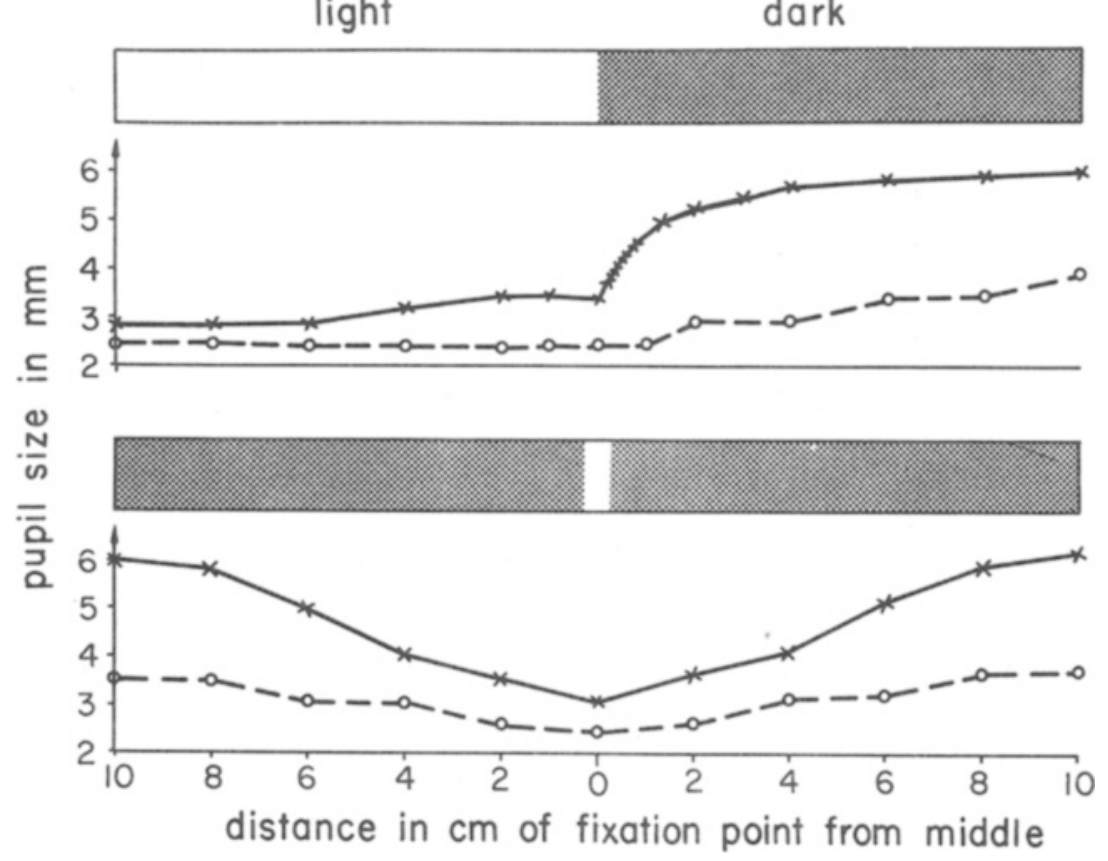




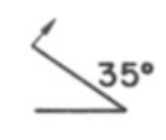

rotation of whole stripe
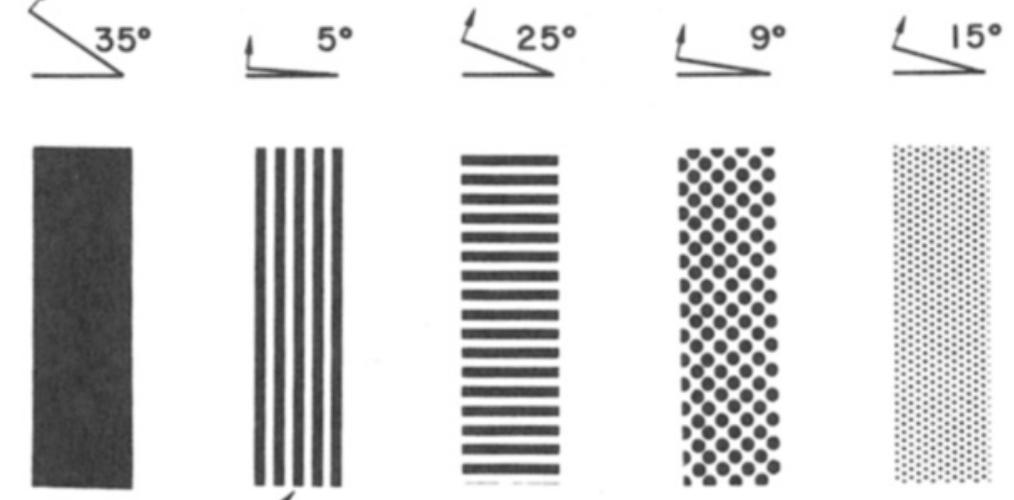

$-12 \mathrm{~cm}-$
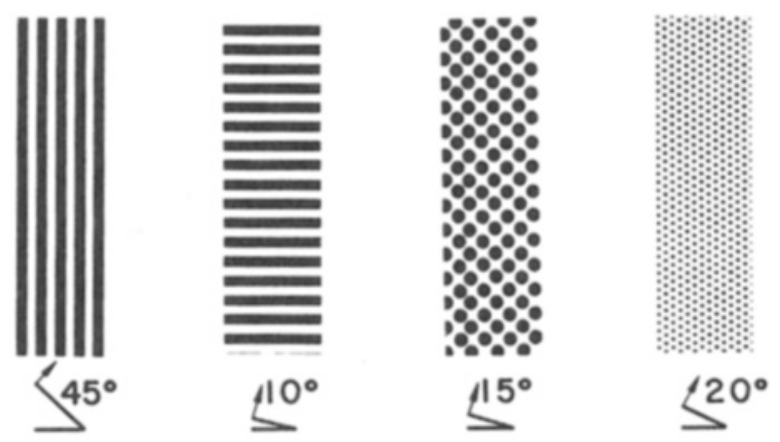

rotation of lines and dots

a snuperscope (USA gunsight for night use). The upper drawing shows how the pupil size increases when the fixation point is moved from the bright side to the darker side of a horizontal stripe. The lower curve shows the same when the fixation point approaches an illuminated white section. The distance of observation was $25 \mathrm{~cm}$ and the luminance of the white surface $10 \mathrm{fL}$. The dashed curves are for an $O$ with decreased mobility of the pupils.

The pupils have the definite tendency to eliminate brightness differences between the eyes for the observed object. But besides this, there is a certain parallel between the movements of the pupils. If, for instance, the left eye is covered with a density filter and the pupil of the left eye increases, the pupil of the right eye will do the same simultaneously but to a lesser degree.

There is even a small range of unbalance in which no compensation between the pupils occurs. For an active pupil with a density filter of 1.0 , the opening of the pupil can be $1 \mathrm{~mm}$ larger than that of the other pupil. The individual differences are very large. One of the surprising observations is that for some people the pupil on one side may stay quite immobile and all the compensation for the pupil unbalance be done only by the other eye.

An interesting question is what happens with people who have a more or less frozen

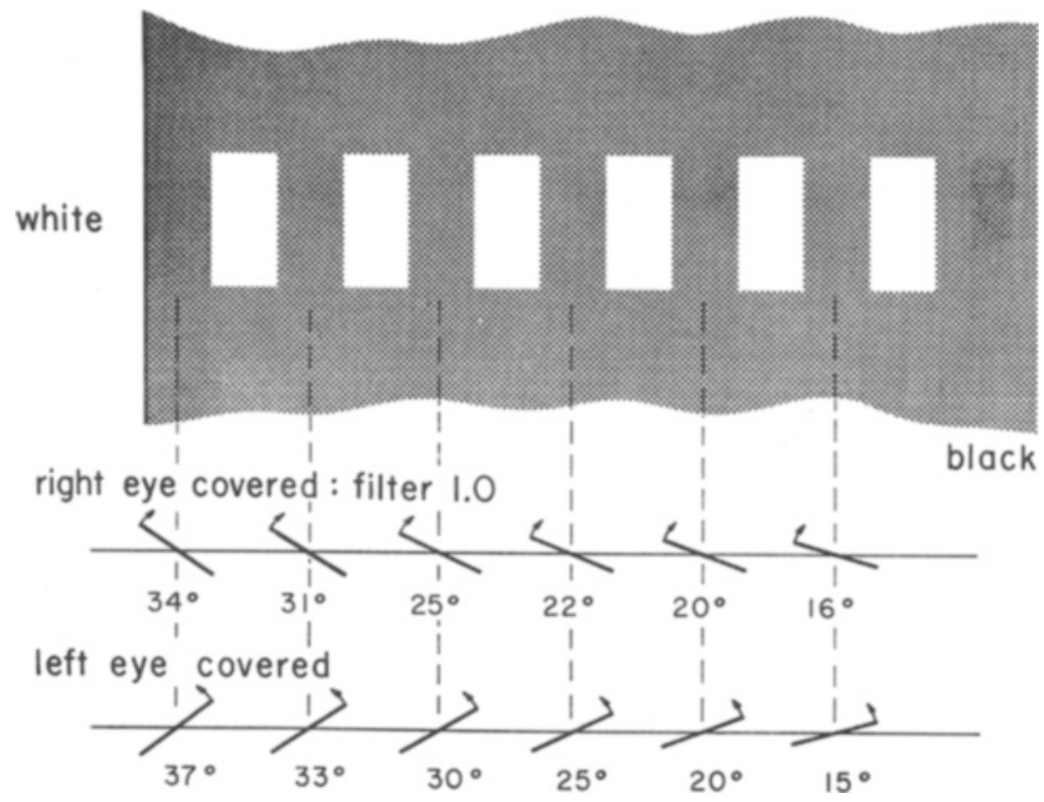

Fig. 7. A vertical stripe made of small black areas shows a rotation as a whole and a rotation of the small black areas. The complex interaction between the two rotations indicates a nervous interaction between neighboring areas.

pupil? In the case I was able to investigate, the $\mathbf{O}$ did see almost everything flat. Since the apparent rotation of an object becomes larger as its size decreases, the difference between a normal $\mathrm{O}$ and one with a frozen pupil became for small objects quite evident. Things looked flat for him because he did see with only one eye. This could be proven when one eye was presented with horizontal stripes and the other eye with vertical ones. There was no fusion and the rivalry between both visual fields was extremely sluggish. The exchange of both eyes occurred maybe in 20 to 40 sec.

Rotation of Complex Luminance Patterns

If the rotation obtained by covering one eye with a density filter is only the consequence of the physical phenomena of irradiation in the optical system of the eye, then we would expect little interaction between two neighboring edges. But this does not seem to be so, indicating that the rotation is strongly influenced by lateral neural processes in the retina or higher up along the central nervous system. The interaction is illustrated in Fig. 7. The width of the five vertical stripes is $2 \mathrm{~cm}$, and they were observed from a distance of $50 \mathrm{~cm}$. When we cover the right eye with a density filter 1.0 , the whole stripe rotates with an angle shown in the upper part of the drawing. The smaller the dots, the larger is their rotation. The same holds for vertical lines. The rotation of the whole stripe with the dots cannot be explained on the basis of the irradiation alone.

The distance for which lateral interaction may occur seems to be quite large. See Fig. 8. The left side of the field of view was white and the right side black, but between the white and the black surfaces was placed a series of white vertical stripes. The white stripes and the surface on the left had a luminance of $5 \mathrm{fL}$. The width of the stripes, black and white, was $2.5 \mathrm{~mm}$, and the apparent rotation of the black stripes when one of the eyes was covered with a density filter 1.0 is illustrated. The more the stripes were located inside the black area, the less rotation. The phenomenon was little

Fig. 8. The rotation of a series of black stripes is essentially modified when a large black area is placed on one end of the series and a white one on the opposite end. 

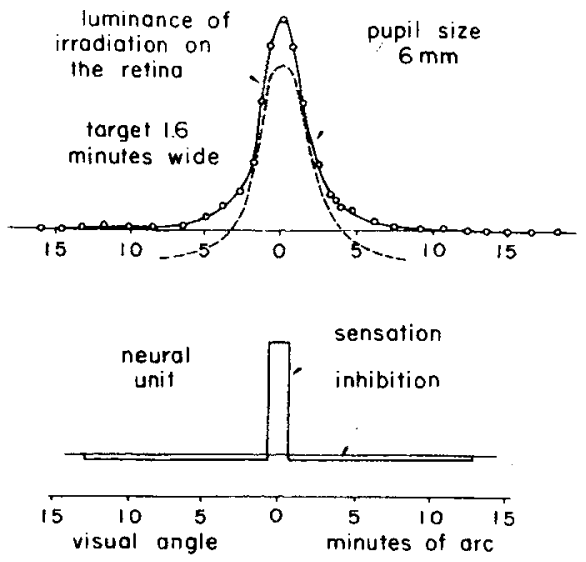

Fig. 9. Irradiation produces a widening of the image on the retina (solid line in the upper drawing) but lateral inhibition seems to narrow the image (dashed line in the upper drawing). The lower drawing shows the neural unit for vision consisting of an area of sensation surrounded by an area of inhibition.

affected by moving the fixation point along the set of stripes. The rotations given in the figure are the data of one $O$, but three other Os had about the same values.

Irradiation vs Mach Bands as the Cause of the Apparent Rotation

It is difficult to decide the relative importance of irradiation and Mach bands, since both produce very similar effects. But there are some differences. If we have a red stripe in a green surrounding and if irradiation is the main feature, then we should be able to observe a white line on the edge, since, by proper selection of the two colors, it is possible to combine them into white. No such white line on the edge was seen.

If the Mach bands are effective in producing the rotation, elimination of the Mach bands should eliminate the rotations also. We can eliminate the Mach bands on the border of two different colors like green and red if we make the brightness equal (Békésy, 1968). When the brightness adjustment between a vertical stripe and its surroundings was done carefully and when one eye was covered with a density filter of 1.0 , no rotation of the stripe was observed. The same results were obtained with colored stripes in a gray surrounding, using three Os.
Many measurements have been done to determine the size of the irradiation produced on the edge of a white stripe (Westheimer \& Campbell, 1962; Krauskopf, 1962; Westheimer, 1963). A summary of retinal image formation is given by Fry (1963). Figure 9 shows in the solid curve of the upper drawing the lateral spread of the luminance on the retina produced by a white stripe of $1.6-\mathrm{min}$ width, according to the measurement of Krauskopf (1962). The lateral spread depends on the pupil's size, which was in this case $6 \mathrm{~mm}$ in diam.

The question of how much this lateral spread is modified by the lateral inhibition has also been discussed recently (Bliss \& Macurdy, 1961; Lowry \& DePalma, 1961; Bryngdahl, 1964; Menzel, 1965; Ratcliff, 1965; Campbell, 1968). Lateral inhibition in the retina and the higher level of the nervous system does seem to reduce the effectiveness of the wide spread of the irradiation, especially for the lower levels of the luminance.

We can illustrate this by applying the earlier-developed concept of a neural unit for vision (Békésy, 1960) to the luminance distribution produced by irradiation shown in the solid curve in the upper drawing of Fig. 9. The lower drawing in Fig. 9 shows the neural unit, which consists of an area of sensation surrounded by an area of inhibition. It is this inhibition which eliminates the influence of the wide lateral spread of the irradiation. To estimate the effect of the lateral inhibition, we can cut the luminance curve of the irradiation in the upper drawing into segments with the width of the sensation area of the unit. Then we adjust the magnitude of the inhibition proportional to the magnitude of the luminance of the irradiation curve for that particular segment. Having done that for all the different segments left and right of the maximum, we add together all the inhibition values for every segment and subtract them from the magnitude of the luminance for that particular segment. The dashed curve in the upper drawing results. The lateral inhibition does increase the steepness of the drop of the sensation on the edge of a white stripe.

This seems to indicate that the apparent rotation produced by a density filter before one eye is a complicated combination of irradiation and lateral nervous interaction similar to that which produces the Mach bands.

\section{REFERENCES}

BÉKÉSY, G. v. Neural inhibitory units of the eye and skin. Quantitative description of contrast phenomena. Joumal of the Optical Society of America, 1960, 50, 1060-1070.

BEKESY, G. v. Brightness distribution across the Mach bands measured with flicker photometry, and the linearity of sensory nervous interaction. Journal of the Optical Society of America, 1968, 58, 1-8.

BLISS, J. C., \& MACURDY, W. B. Linear models for contrast phenomena. Journal of the Optical Society of America, 1961, 51, 1373-1379.

BRYNGDAHL, O. Visual transfer characteristics from Mach band measurements, Kybernetik, 1964, 2, 71-77.

CAMPBELL, F. W. The human eye as an optical filter. Proceedings of the IEEE, 1968, 56, 1009-1014.

CIBIS, P. A., \& HABER, H. Anisopia and perception of space. Journal of the Optical Society of America, 1951, 41, 676-683.

DAVSON, $\mathrm{H}$. The physiology of the eye. Philadelphia: Blakiston, 1950.

FRY, G. A. Retinal image formation: Review, summary, and discussion. Journal of the Optical Society of America, 1963, 53, 94-97.

KRAUSKOPF, J. Light distribution in human retinal images. Journal of the Optical Society of America, 1962, 52, 1046-1050.

LOWRY, E. M., \& DePALMA, J. J. Sine-wave response of the visual system. I. The Mach phenomenon. Journal of the Optical Society of America, $1961,51,740-746$.

MENZEL, E. Der Gesichtssinn als linearer Übertragungskanal und die Machschen Streifen. Die Naturwissenschaften, 1965, 55, 316-317.

MÜNSTER, C. Über den Einflun von Helligkeits Unterschieden in beiden Augen auf die stereoscopische Wahmehmung. Zeitschrift für Sinnesphysiologie, 1941, 69, 245-260.

OGLE, K. N. The optical space sense. In $H$. Davson (Ed.), The eye. Vol. 4. New York: Academic Press, 1962. Pp. 302-303.

OGLE, K. N. Researches in binocular vision. New York and London: Hafner, 1964.

RATLIFF, F. Mach bands: Qualitative studies on neural networks in the retina. San Francisco, London, and Amsterdam: Holden-Day, 1965.

WESTHEIMER, G. Optical and motor factors in the formation of the retinal image. Joumal of the Optical Society of America, 1963, 53, 86-92.

WESTHEIMER, G., \& CAMPBELL, F. W. Light distribution in the image formed by the living human eye. Journal of the Optical Society of America, 1962, 52, 1040-1045.

\section{NOTES}

1. The work has been supported by the National Institutes of Health (Grant NB-06890). 2. Address: Laboratory of Sensory Sciences, University of Hawaii, 1933 East-West Road, Honolulu, Hawaii 96122.

(Accepted for publication January 28, 1970.) 